\title{
La doctoresse Charlotte Olivier (1865-1945) et la prise en charge des tuberculeux indigents à Lausanne
}

par Geneviève Heller

Résumé

La tuberculose, maladie contagieuse aggravée par la promiscuité et la misère, est considérée au début du $20^{e}$ siècle comme une maladie sociale. La thérapie étant aléatoire jusqu'à la découverte des antibiotiques, une prévention indirecte semble s'imposer (réseau d'œuvres, conditions de logement, protection de l'enfance, etc.). On assiste au développement d'une médecine sociale et préventive qui prend en considération le malade et son milieu. L'intrusion dans la vie privée est le corollaire de l'assistance. Ce sont principalement des femmes qui prennent part à la lutte contre la tuberculose: non professionnelles, infirmières-visiteuses et médecins. Parmi ces dernières, un rôle particulièrement important est joué par Charlotte Olivier, qui dirige le Dispensaire antituberculeux de Lausanne de 1911 à 1925 .

Lorsque l'on évoque la tuberculose du début du siècle, on s'attend peut-être à ce que l'on parle des sanatoriums, et en particulier pour le Canton de Vaud, de la Ville sur la montagne qu'était alors Leysin ${ }^{1}$, réputée bien au-delà de nos frontières. La tuberculose dont il est question ici est plus particulièrement celle du peuple. Non que la tuberculose des pauvres fût différente de celle des riches étrangers qui venaient se faire soigner à Leysin, mais c'est l'approche médicale et sociale qui a été différente. Ce n'est pas une médecine d'élite pratiquée par des hommes, mais une médecine sociale s'appuyant essentiellement sur des femmes. C'est du moins le cas dans le canton de Vaud ${ }^{2}$. La principale actrice à Lausanne était la doctoresse Charlotte Olivier, précédée et assistée de quelques hommes, mais secondée par un véritable réseau de femmes.

Trois domaines seront évoqués ici :

Une brève introduction donne quelques points de repère concernant le traitement de la tuberculose qui devraient permettre de mieux appréhender la situation des tuberculeux indigents.

Puis l'intervention de la Doctoresse Charlotte Olivier qui a tenté de mettre sur pied un réseau d'action et de prévention rayonnant à partir d'un 
Dispensaire, s'ouvrant vers des institutions coordonnées entre elles pour prendre en charge les tuberculeux indigents. On assiste ainsi au développement d'une médecine sociale et préventive.

Enfin, pour mettre sur pied une telle médecine, déconsidérée dans le contexte de l'époque, il a fallu mobiliser des bonnes volontés, des femmes principalement, et former un personnel adéquat, les infirmières-visiteuses, professionnelles de l'assistance.

\section{Une maladie ruineuse}

La tuberculose était dans la deuxième moitié du $19^{\text {e }}$ siècle une maladie bacillaire très répandue, le plus souvent fatale lorsqu'elle se développait; contractée généralement dans l'enfance, elle ne se manifestait souvent que plus tard, lorsque des circonstances affectaient l'équilibre précaire de l'organisme; ce fut le cas des soldats pendant la guerre. La tuberculose pulmonaire ou phtisie, la plus répandue et la plus dangereuse des formes tuberculeuses, touchait particulièrement les forces actives de la société, les jeunes gens et les jeunes filles, les travailleurs, les pères et mères de famille: la moitié des décès dans la population âgée de 18 à 40 ans était due, vers 1900, à la tuberculose.

Il n'existait aucun médicament ou traitement spécifique. Les pionniers Herrmann Brehmer et son élève Peter Dettweiler, fondateurs de la cure sanatoriale, préconisèrent dans les années 1850-1880 un traitement hygiénique global pour les tuberculeux: le repos, une saine alimentation et une aération régulière. Ceci correspondait à un renouveau de la médecine naturelle.

La cure sanatoriale visait au fond à mettre au repos tout l'organisme. Peu à peu s'est développée, dès le début du $20^{\text {e }}$ siècle, une thérapie de la tuberculose pulmonaire, visant à mettre au repos non seulement tout l'organisme de manière indifférenciée, mais plus particulièrement l'organe malade en intervenant localement comme par exemple en pratiquant un pneumothorax ${ }^{3}$ ou une thoracoplastie ${ }^{4}$. Cette thérapie s'ajoutait généralement à la cure sanatoriale passive, lui offrant plus de succès, succès tout relatif d'ailleurs. Ce n'est qu'après la Deuxième Guerre mondiale que l'on a appliqué des traitements chimiothérapiques efficaces condamnant au repos le bacille luimême (sans le détruire totalement), grâce en particulier à la découverte de la streptomycine.

D'autre part la tuberculose pulmonaire était particulièrement contagieuse. La contagiosité de la maladie, soup̧̧onnée mais généralement déniée 
par une partie du corps médical, avait été attestée en 1882 par Robert Koch qui en a découvert le bacille. La règle du sanatorium était, en principe, stricte, et l'éloignement de la famille nécessaire. La cure était de très longue durée, des mois, généralement des années.

\section{Une cure impossible}

On peut imaginer dès lors le désastre matériel (sans parler du désastre individuel commun aux riches et au pauvres) dans lequel se trouvaient les tuberculeux indigents et leur famille. Le sanatorium ne pouvait être qu'une solution très partielle dans leur prise en charge.

Il faut noter que, au début du siècle, le succès des cures sanatoriales était très relatif, rechutes et aggravations étaient la règle surtout pour ceux qui redescendaient dans le monde. En 1920, une enquête courageuse portant sur 1200 malades hospitalisés 5 ans plus tôt au Sanatorium populaire révèle que $45 \%$ de ces malades sont morts entre-temps. Il faut ajouter qu'à cette époque, le traitement en sanatorium pour la grande masse des tuberculeux était une exception. Certes, il y avait des sanatoriums populaires ${ }^{5}$ pour les malades peu fortunés ou à charge de l'Etat, mais le nombre de lits à disposition était dérisoire en comparaison des cas que l'on commençait à repérer. D'autre part, les bénéfices de la cure disparaissaient rapidement avec les conditions de vie et de travail de cette population laborieuse.

Cette brève introduction situe le contexte dans lequel Charlotte Olivier va intervenir : il fallait trouver d'autres moyens d'aborder la tuberculose des indigents pour lesquels une hospitalisation de très longue durée était pour ainsi dire inaccessible, leurs familles ruinées et épuisées, les rechutes fatales presque inévitables, tandis que la contagion poursuivait son chemin inexorablement. D'autre part, si la tuberculose se rencontrait dans toutes les couches de la société, il est bien évident qu'une population affaiblie par la malnutrition, l'épuisement du travail, l'alcoolisme et spatialement très rapprochée de par la promiscuité des conditions d'habitation, cette population-là était plus vulnérable.

La situation était ainsi dramatique économiquement pour les familles touchées bien sûr, mais plus globalement pour la société qui avait à charge les malades et leurs proches et qui perdait du même coup une main d'œuvre utile; la situation était en plus, dans le cas des masses contagieuses, dangereuse pour la société. En Suisse, les efforts avaient été concentrés principale- 
ment sur quelques cités d'en-haut (Davos, Montana, Leysin), alors que presque rien n'était fait pour la population dans les villes et à la campagne. Depuis 1887, Sir Robert Philip à Edimbourg et, depuis 1901, Calmette à Lyon, avaient donné la preuve que l'expansion de la maladie pouvait être maîtrisée grâce à une prévention active. En Suisse rien de tel encore.

\section{Biographie de Charlotte Olivier}

C'est ici qu'intervient le cas présenté aujourd'hui. Il s'agit d'une approche à travers une biographie ${ }^{6}$, celle de Charlotte Olivier (1865-1945), médecin du Dispensaire antituberculeux de Lausanne pendant les années déterminantes entre 1911 et 1925, puis médecin-conseil de la Ligue vaudoise contre la tuberculose et responsable en particulier des infirmières-visiteuses.

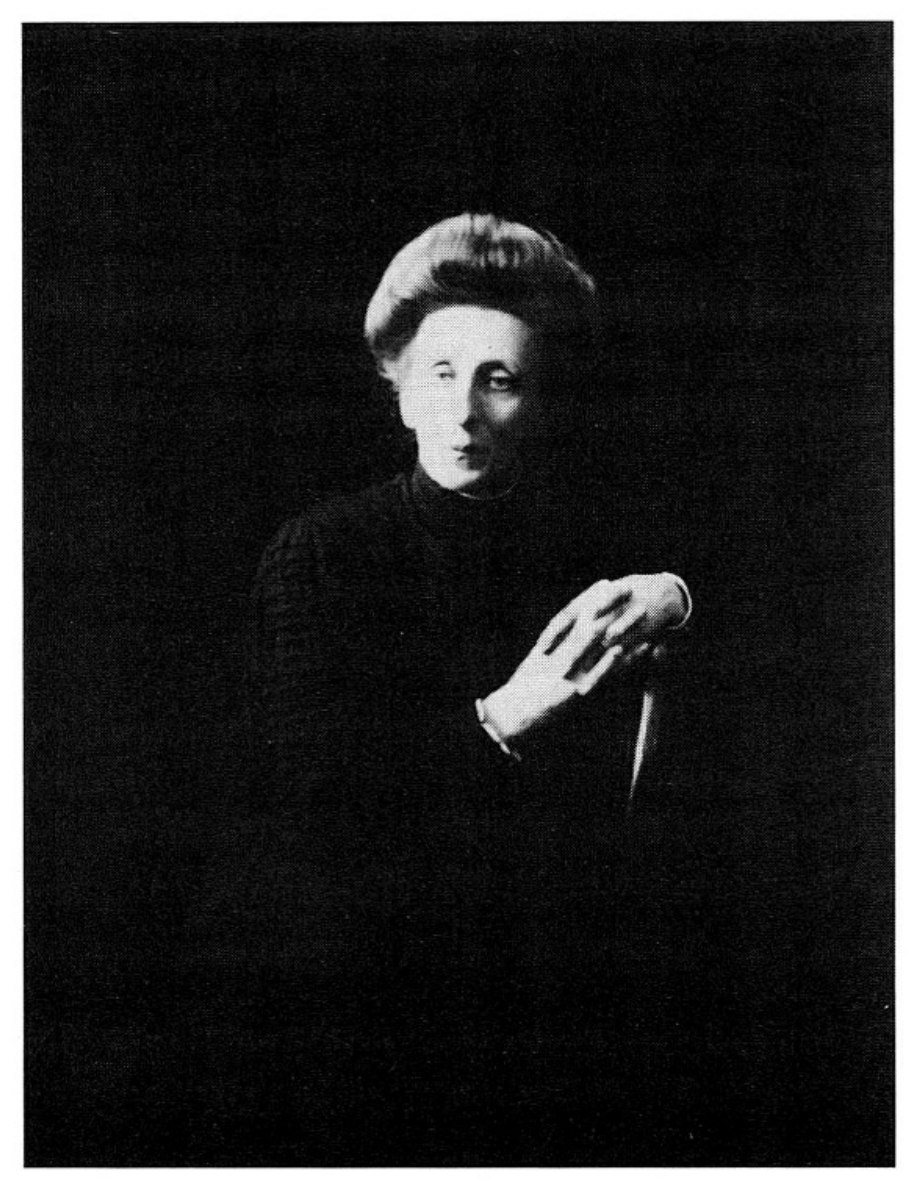

Charlotte Olivier-von Mayer, 1912. Photo de Jongh, Musée historique de Lausanne. 
L'origine particulière de Charlotte Olivier-von Mayer a sans aucun doute donné le ton, l'énergie, l'arrogance pour ainsi dire, de ses interventions dans une région où la mentalité est plutôt à la retenue et à l'expectative. Elle est née à Saint-Pétersbourg où elle a vécu jusqu'à ses études de médecine faites à Lausanne à l'âge de trente ans; son milieu est d'origine germanique et évangélique - c'est-à-dire protestant -, très imprégné du Réveil religieux qui s'est développé au début du $19^{\mathrm{e}}$ siècle. Son père, médecin lui-même, avait fondé un hôpital à Saint-Pétersbourg formant son personnel - des diaconesses - et accueillant principalement des indigents. La tuberculose était familière à Charlotte Olivier qui avait vu les nombreux patients de son père. Enfin, elle a côtoyé la maladie de son mari tuberculeux, Eugène Olivier; médecin lui-même, secrétaire de la Ligue vaudoise contre la tuberculose entre 1913 et 1926, il a voué la seconde partie de sa vie à l'histoire de la médecine dans le Canton de Vaud. Il fut sans aucun doute un partenaire indispensable de la campagne menée activement par sa femme.

La vocation de Charlotte Olivier apparaît comme une douloureuse mais impérative nécessité. Son mari écrira:

«C'est pour libérer sa conscience et soulager son cœur qu'elle s'est mise en route dans cet apostolats ${ }^{7}$.

Sa vie apparaît totalement austère, mais son acharnement (presque un vice) a eu raison de la torpeur ambiante.

\section{Une médecine sociale et préventive}

Le Dispensaire antituberculeux de Lausanne dont Charlotte Olivier fut responsable, a été fondé en 1906, soit une année après le Congrès international de la tuberculose de Paris. La même année sont ouverts les dispensaires de Genève et de Neuchâtel.

Un dispensaire antituberculeux est, plus que toute autre consultation des pauvres, indispensable pour une ville. Charlotte Olivier s'en explique:

«D'abord, en opposition aux autres maladies contagieuses, qui sont de courte durée, la tuberculose est une maladie essentiellement chronique; il faut pour la guérir un an, deux ans, six ans.

Ensuite, la tuberculose est un mal qui ne se combat pas par la simple distribution de médicaments. Elle exige de celui qui en est atteint toute une rééducation, parfois une refonte entière de sa manière de vivre; le tuberculeux a pour cela besoin d'être dirigé. 
Enfin, et ceci est le point capital, la tuberculose est entretenue par les conditions d'existence que l'on peut désigner d'un mot par ce terme: la misère sociale.

Ainsi, pour lutter avec succès contre la tuberculose, il faut beaucoup de temps, beaucoup d'efforts personnels, beaucoup d'argent.

Est-il encore besoin d'ajouter que les milliers de malades de la classe ouvrière sont hors d'état de satisfaire par eux-mêmes à ces exigences? Même dirigés par le médecin le plus instruit et le plus dévoué, ils n'y arrivent pas. Le Dispensaire est né de ces conditions mêmes; il est l'organe adapté à combattre la tuberculose-maladie populaire» .

Les fonctions du D.A.T. sont largement orientées vers une prise en charge sociale: en plus des consultations gratuites, le dispensaire assure des soins médicaux à domicile, le dépistage des nouveaux cas, la surveillance des enfants et du logement, l'hospitalisation et le placement des tuberculeux, l'assistance alimentaire, l'éducation à l'hygiène, etc.

Le dispensaire procure des bons alimentaires (œufs, lait, viande, farine enrichie de phosphate). Il apporte une aide matérielle (un secours pour payer le loyer, éventuellement la recherche d'un logement plus convenable). Il procure un lit pour que le tuberculeux puisse dormir seul. Pour soulager une mère, le linge est lavé, désinfecté, réparé, par les soins du dispensaire et d'un réseau de dames qui apportent leur aide bénévole. En outre, le Dispensaire met à disposition de ses patients une cure d'air, sorte d'abrégé de la cure sanatoriale, pour quelques heures de repos fortifiant.

\section{Objectifs du dispensaire}

Les soins du D.A.T. consistent moins à traiter les lésions pulmonaires ou d'autres lésions tuberculeuses, qu'à fortifier les patients, à «améliorer leur état général, à augmenter leur capacité de travail et leur résistance. Pour un tuberculeux pauvre, tout est là» ${ }^{9}$. Il est bien question ici de prolonger, de «faire durer» ${ }^{10}$ autant que possible la vie active de ceux qui ont une charge familiale.

«Nous maintenons ainsi, dans bien des cas, l'équilibre nécessaire, physique autant que moral, qui permet à l'ouvrier, à la mère de famille malade, mais indispensable aux siens, de ne pas sombrer prématurément» ${ }^{11}$.

Si l'on tente de conserver pour un temps la force de travail d'un père de famille ou la présence au foyer d'une mère, ce n'est pas pour leur profit ou leur bonheur personnel, car de telles vies sont gâchées par l'épuisement, c'est bien un maillon de l'ordre social que l'on tente de sauver pour un temps qui, 


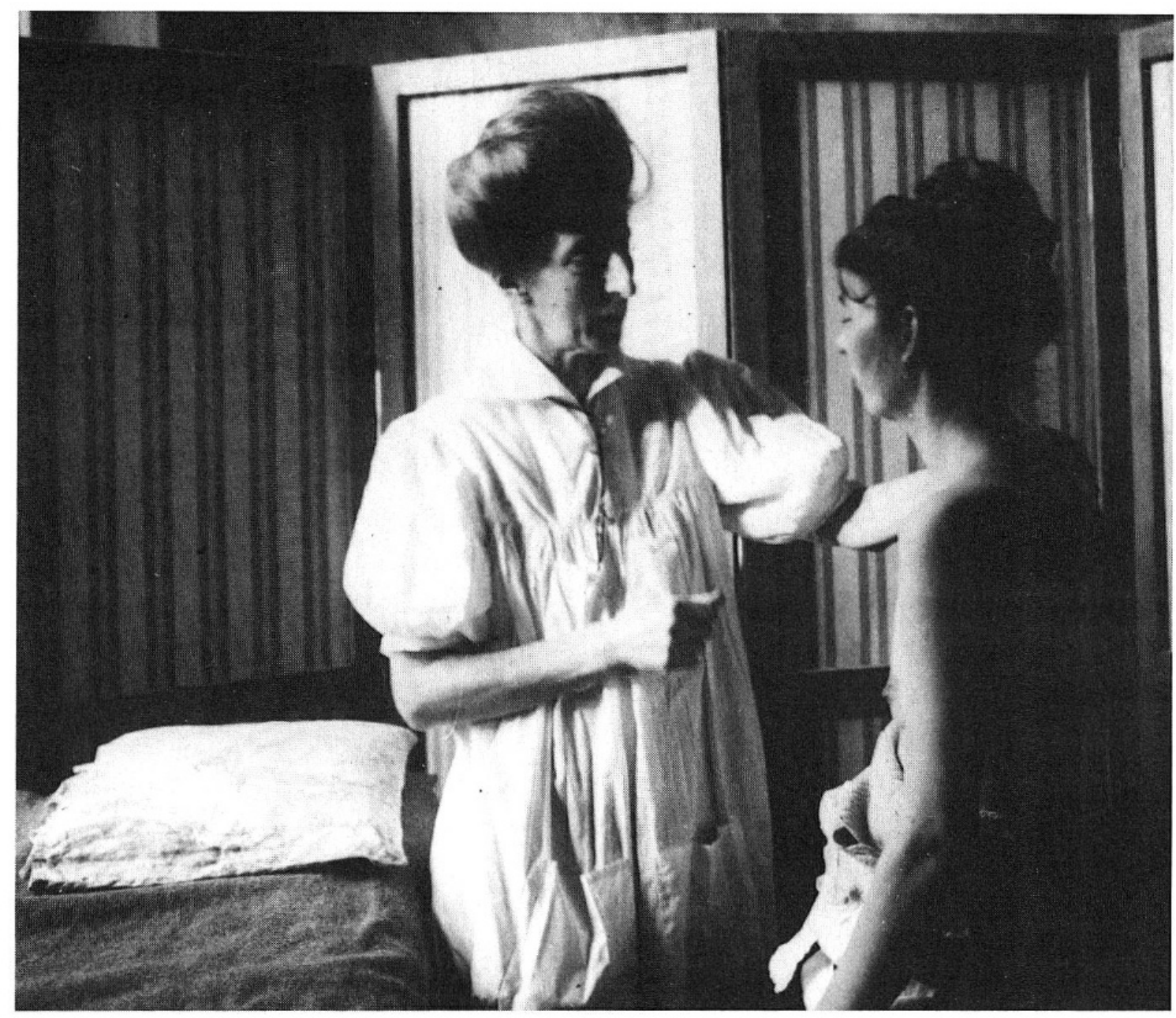

La doctoresse Charlotte Olivier-von Mayer avec une patiente au Dispensaire antituberculeux de Lausanne en 1917. Musée historique de Lausanne.

multiplié par 10 ou 20, ou 200, représente une économie pour la société. C'est un premier objectif.

Un second objectif, plus important encore, est de limiter la contagion: il s'agit, en prenant soin d'un tuberculeux, de surveiller son entourage et de repérer ceux qui ont été contaminés. Le dépistage est fondamental. Cette investigation doit se faire en amont et en aval, remonter la filière de l'infection et suivre désormais ceux des proches, les enfants en particulier qui ne sont peut-être pas malades, mais prédisposés.

Parallèlement il faut construire, brique après brique, les différents éléments de l'édifice antituberculeux : toutes sortes de lieux seront peu à peu mis à disposition pour héberger les tuberculeux assistés ou les membres de leur famille, des fonds divers seront exploités et sollicités pour subvenir aux 
dépenses le temps de leur traitement, des compromis seront sans cesse cherchés pour choisir la moins mauvaise solution dans les conditions précaires où se trouvent la plupart des malades du Dispensaire.

De plus en plus on cherche à repérer et à hospitaliser les tuberculeux au premier degré pour lesquels une cure est réellement bénéfique. Les enfants font l'objet d'efforts tout à fait particuliers, soit pour les soigner, soit pour prévenir la contagion de leur milieu infecté. On ouvre des préventoriums pour les enfants pas encore ou peu contaminés qu'il faut isoler de leurs parents. Disloquer les familles est une opération extrêmement délicate et dont le bien-fondé est difficile à faire comprendre. Dans le catalogue des œuvres antituberculeuses, on crée aussi des cures d'air, des colonies de vacances. Les enfants sont surveillés par le service médical scolaire. Les conditions d'habitation et l'hygiène du logement sont contrôlés plus sévèrement. Enfin, on se donne tardivement (en 1928) les moyens légaux permettant de forcer les récalcitrants en même temps que la Confédération accorde une participation financière aux œuvres antituberculeuses ${ }^{12}$. Le dispensaire apparaît donc comme le centre d'un réseau d'actions complémentaires.

\section{Intrusion révélatrice}

"[Cependant] l'aide du dispensaire se fait à l'unique condition d'accepter la surveillance du domicile et des enfants. [...] Nous estimons inadmissible d'accorder ces subsides à une famille, aussi longtemps qu'elle s'obstine à vivre d'une façon peu hygiénique. [...] L'intrusion dans la famille est [...] la condition indispensable de toute lutte antituberculeuse efficace» ${ }^{13}$.

La tuberculose est, au sein de la population indigente, comme un fil d'Ariane qui conduit au cœur de la misère sociale. Aucun argument, aucun prétexte ne pouvait justifier une ingérence dans les foyers mieux que la lutte contre une maladie si épuisante pour le malade et si dangereuse pour son entourage. La population se trouve pour ainsi dire acculée à dévoiler sa misère. La lutte contre la tuberculose devient nécessairement une lutte contre tout ce qui lui est associé dans ce contexte: alcoolisme, pauvreté, chômage, enfance malheureuse, logements insalubres, etc.

La soumission exigée des patients du Dispensaire n’est donc pas simple docilité en échange des bons soins accordés, elle est un droit de regard sur le réseau de la tuberculose et de la misère sociale. Une fois repérés par le 
Dispensaire, le malade indigent et son milieu allaient être assistés et réformés.

\section{Le rôle des femmes}

L'expérience du Dispensaire devait se généraliser dans toute la région. C'est alors que Charlotte Olivier s'est adressée en 1912 à l'Union des femmes, une organisation de dames bénévoles, instruites, motivées et ayant du temps à disposition. Pour les sensibiliser elle donne des exemples de situations dramatiques, révoltantes, dangereuses.

«Mesdames [leur dit-elle], devinez-vous maintenant votre champ d'activité, au milieu de ce défilé d'ombres que j'ai fait rapidement passer devant vos yeux? Avouez-le, que peut faire le meilleur médecin, en face de ces conditions anti-hygiéniques révoltantes? ${ }^{14}$.

Est-ce lui qui trouvera le lit pour le tuberculeux, l'argent pour un meilleur logement, qui se chargera de l'éducation antituberculeuse des parents, qui protègera les enfants?

«Non, Mesdames, tout cela est de votre domaine.» ${ }^{15}$

Il faut sensibiliser un maximum de femmes pour installer dans chaque localité des comités actifs. Toute la campagne est basée sur une information rayonnante, à diffusion aussi large que possible, mais toujours personnelle, de femme à femme. Elles sollicitent de nouveaux membres, organisent des collectes, des conférences, confectionnent et réparent le linge, font des visites à domicile.

\section{Profession: infirmière-visiteuse}

Assez vite on se rend compte que l'intervention des dames ne répond plus aux exigences de cette exploration systématique et de longue durée des familles contaminées. La bonne volonté, même bien informée, ne suffit plus. Les exigences se professionnalisent. Il faut réunir cohérence et continuité. Un métier se dessine.

Les nouvelles compétences de l'infirmière antituberculeuse se précisent, distinctes de celles de la garde-malade. La différence fondamentale est que l'infirmière-visiteuse ne s'occupe pas à proprement parler de la maladie du 
tuberculeux, mais se soucie de son entourage ainsi que des conditions matérielles et sociales dans lesquelles ils se trouvent, lui et sa famille.

«Introduite dans le milieu, [l'infirmière-visiteuse] se met à l'assainir. Son premier devoir n'est pas de donner des soins, d'agir en garde-malade dans le sens usuel du mot; pour l'infirmière-visiteuse, le tuberculeux malade est avant tout membre d'une collectivité, victime des torts de son entourage. Elle doit l'empêcher par tous les moyens dont dispose l'hygiène actuelle de nuire à d'autres à son tour.» ${ }^{16}$

C'est un métier polyvalent, en théorie une sorte d'apothéose de la profession féminine. L'infirmière-visiteuse devient une professionnelle de l'enquête et de l'assistance, elle est la cheville ouvrière de tout un réseau d'œuvres et d'institutions qui ont à faire aux tuberculeux et à leur famille. Elle sera peu à peu remplacée, vers 1950, par l'assistante sociale.

«Eloigner si possible le malade contagieux; l'isoler au milieu des siens si l'hospitalisation ne peut être obtenue, attaquer les causes secondaires qui entretiennent la tuberculose: la saleté, le manque d'air, le logis insalubre; faire procéder au déménagement dans un logis plus sain; trouver, dans ce but, les moyens financiers nécessaires; s'occuper de la famille pendant que père ou mère sont hospitalisés; trouver un travail adéquat à ceux qui rentrent du sanatorium incomplètement guéris. Surtout, et c'est là le nœud de la question, le couronnement de tout ce travail: elle doit dépister les nouveaux cas de tuberculose; faire examiner tous les membres de la famille avant qu'ils soient vraiment malades, surveiller les enfants, enseigner l'hygiène, donner des conseils, encourager, se faire aimer et ne plus lâcher cette famille jusqu'à ce que tout danger soit écarté. Il faut avoir assisté à cette tâche ou y avoir mis la main soi-même pour savoir combien elle est lourde, combien souvent elle échoue, mais combien aussi elle seule signifie lutte contre la tuberculose, maladie familiale, maladie sociale». ${ }^{17}$

Nous avons ici quelques éléments expliquant pourquoi une large part de la lutte contre la tuberculose a été assumée par des femmes. L'impuissance de la médecine à l'égard de la tuberculose en faisait une maladie dévalorisée, c'était une maladie gênante dans le tableau de chasse d'une médecine triomphante. Cette médecine sociale et préventive n'était pas l'apanage des hommes-médecins, occupés à des tâches plus nobles, plus scientifiques, plus techniques (la chirurgie thoracique était une exception dans le cas de la tuberculose). Charlotte Olivier a reproché plus d'une fois que l'on soignait l'organe à Leysin, renvoyant le malade totalement déclassé dans son milieu. On ne reconnaissait pas encore une valeur médicale à l'hygiénisme, ni au sein du corps médical, ni à plus forte raison dans la population.

Mais cela convenait si bien aux femmes et à leur vocation ancestrale de dévouement, d'éducation, de service du prochain: c'était bon pour les femmes de se pencher sur tant de problèmes matériels et psychologiques. De 
même que l'on avait découvert depuis peu l'importance du métier de gardemalade - indispensable au succès de la médecine hospitalière -, on allait découvrir l'importance de l'assistance psychologique et sociale des maladies de longue durée, complément primordial mais combien délicat au traitement médical. On peut ajouter une autre explication, complémentaire des autres, à la prédominance féminine. Peu prisée par le corps médical, la lutte contre la tuberculose offrait un champ d'action pour ainsi dire libre, à disposition des femmes-médecins qui sans aucun doute pénétraient difficilement dans le cénacle médical. Revenons à l'exemple de Charlotte Olivier: interne en chirurgie chez César Roux, très appréciée de lui et selon toute vraisemblance excellente chirurgienne, elle n'a pas poursuivi dans ce domaine. Elle a fait un autre choix, dans les circonstances qui se sont présentées à elles.

La part prise par Charlotte Olivier est largement inspirée et soutenue par une éthique rigoriste et exigeante. La lutte contre la tuberculose était une façon de corriger le monde ${ }^{18}$; c'est une prévention qui a largement dépassé ses objectifs en étant un instrument privilégié de réforme sociale. 


\section{Notes}

1 Je renvoie ici à mon article paru dans Gesnerus 47, 1990, Leysin et son passé médical, p. 329-344.

2 Le modèle de référence fut celui de Sir Robert Philip, fondateur du dispensaire antituberculeux d'Edimbourg en 1887.

3 Insufflation d'air dans la cavité de la plèvre (entre poumon et thorax), réduisant le mouvement respiratoire et permettant de mettre au repos les parties malades du poumon. La première application est due au médecin italien Carlo Forlanini en 1894, elle ne fut adoptée qu'au début du siècle suivant.

4 Opération, inaugurée en 1885, consistant à enlever, en les sciant, une partie des côtes se trouvant au niveau de la lésion pulmonaire. La paroi thoracique s'affaisse permettant au poumon de se rétracter et de ne plus travailler à cet endroit.

5 Celui de Leysin fut inauguré en 1902, pouvant accueillir une centaine de patients non fortunés (mais pas démunis), l'Etat de Vaud prenant à sa charge vingt lits pour indigents.

6 Mandat de recherche de l'Institut universitaire d'histoire de la médecine et de la santé publique à Lausanne, 1989-90.

7 Olivier Eugène, Biographie dactylographiée, p. 1 [Bibliothèque cantonale et universitaire de Dorigny-Lausanne, Cabinet des manuscrits, IS 1905/214].

8 [Olivier Charlotte], Rapport du Dispensaire antituberculeux de la Policlinique universitaire pour 1917, Lausanne, p. 4 [rétrospective des dix premières années du Dispensaire].

9 Idem, 1911, p. 43.

10 Idem, 1913, p. 11.

11 Idem, 1911, p. 44.

12 Loi fédérale sur la lutte contre la tuberculose du 13 juin 1928. La loi est le résultat de douze projets successifs depuis 1913. L'intervention de Charlotte Olivier a été décisive. Elle avait déjà obtenu du Président de la Confédération, Ernest Chuard, l'arrêté du 22 mars 1922 accordant un million de francs par année à la lutte contre la tuberculose.

13 Rapport du Dispensaire antituberculeux, 1914, p.11.

14 Olivier Charlotte, La lutte contre la tuberculose, in Bulletin féminin, 1912, p. 51.

15 Idem, p. 51.

16 Olivier Charlotte, Le médecin et l'infirmière-visiteuse dans la lutte contre la tuberculose, in Contre la tuberculose, 12, 1921, tiré à part, p. 9 [exposé à l'Assemblée générale de l'Association suisse contre la tuberculose].

17 Idem, p. 3.

18 S'opposant ainsi au Misanthrope de Molière:

«Et c'est une folie à nulle autre seconde

De vouloir se mêler de corriger le monde», [Le Misanthrope, I, 1.] 


\section{Références bibliographiques}

Gottraux-Biancardi, Helena, L'âge d'or de la Ligue vaudoise contre la tuberculose, Certificat de politique sociale à la Faculté des sciences économiques et sociales de l'Université de Genève, 1987, dactylographié.

Grellet, Isabelle, \& Kruse Caroline, Histoires de la tuberculose, Les fièvres de l'âme 1800-1940, Paris, 1983.

Heller, Geneviève, Charlotte Olivier. La lutte contre la tuberculose dans le canton de Vaud, Lausanne, 1992.

Nicati, Marthe, $B K+$, le prix de la guérison, Lausanne, [Payot], 1950.

Olivier, Charlotte, La lutte contre la tuberculose, [travail présenté à l'Assemblée cantonale de l'Union des femmes de Lausanne, le 29 mai 1912], in Bulletin féminin, juin 1912, p.45-55.

[Olivier, Charlotte], Dispensaire antituberculeux de la Policlinique universitaire, Cure d'air de Sauvabelin, Lausanne, Rapports annuels de 1911 à 1926.

Saudan, Guy, Dispensaire et policlinique à Lausanne: 100 ans de médecine et société, in Revue médicale de la Suisse romande 109, 1989, p. 267-277.

Vallotton, Benjamin, Charlotte Olivier, Contre vents et marées 1864-1945, Lausanne, 1948.

Vincent, Jules, Gardienne de ses frères, Charlotte Olivier, Lausanne, 1954. 


\section{Summary}

Dr Charlotte Olivier (1865-1945) and the care for the tuberculous poor at Lausanne

At the beginning of the 20th century, tuberculosis is regarded as a social disease. For lack of an anti-infectious therapy, an indirect prophylaxis seems mandatory: a network of institutions, sanitation of dwellings, protection of children. One assists at the development of a social and preventive medicine which cares for the sick in their social environment. This implies, however, intrusion into private life. Fighters against tuberculosis in this form are predominantly women: charitable ladies, visiting nurses, and doctors. Among the latter, Charlotte Olivier is prominent; in the decisive years of 1911-1925 she directs the Lausanne antituberculous dispensary.

\section{Zusammenfassung}

\section{Dr.med. Charlotte Olivier (1865-1945) und die Fürsorge für arme Tuberkulöse in Lausanne}

Die Tuberkulose gilt zu Beginn des 20.Jahrhunderts als soziale Krankheit. Mangels einer antiinfektiösen Therapie scheint sich die indirekte Prophylaxe aufzudrängen: ein Netz von Sozialwerken, Sanierung der Wohnverhältnisse, Schutz der Kinder. Man beobachtet den Aufbau einer Sozial- und Präventivmedizin, die sich um die Kranken in ihrem Milieu kümmert. Das Eindringen in das Privatleben ist die Begleiterscheinung der Fürsorge. Der Kampf gegen die Tuberkulose wird in dieser Form vor allem von Frauen geführt: Laienhelferinnen, ambulante Krankenschwestern, Ärztinnen. Unter letzteren ragt Charlotte Olivier heraus, die in den entscheidenden Jahren 1911-1925 das Dispensaire antituberculeux in Lausanne leitet.

Geneviève Heller, Dr ès lettres, Historienne

Rue du Signal 17

1018 Lausanne 